\title{
Produção de serrapilheira no Cerrado e Floresta de Transição Amazônia-Cerrado do Centro-Oeste Brasileiro
}

Carlos José da SILVA ${ }^{1}$; Luciana SANCHES², Monica Elisa BLEICH${ }^{1}$, Francisco de Almeida LOBO$^{2}$, José de Souza NOGUEIRA ${ }^{2}$

\section{RESUMO}

(Produção de serrapilheira no Cerrado e Floresta de Transição Amazônia-Cerrado do Centro-Oeste Brasileiro). O presente trabalho teve como objetivo verificar a variação da produção de serrapilheira de diferentes biomas: Cerrado (com as fitofisionomias Cerrado Sensu stricto e Cerradão) e Floresta de Transição Amazônia-Cerrado, em clima tropical. Para a determinação da produção de serrapilheira foram utilizados coletores de tela em náilon. Dados micrometereológicos foram coletados nas áreas de estudo. A produção de serrapilheira nos dois biomas mostrou acentuada sazonalidade, com as maiores produçôes ocorrendo durante a estação seca e menor durante a estação chuvosa. A maior produção de serrapilheira ocorreu na Floresta de Transição, seguida do bioma Cerrado. A fração de folhas foi mais representativa do que as frações de galhos, flores, frutos em ambas as áreas estudadas.

PALAVRAS-CHAVE: Serrapilheira, Cerrado sensu stricto, Cerradão, Floresta de Transição Amazônia-Cerrado.

\section{Litterfall production in the Brazilian mid-western Amazonia-Cerrado transition forest}

\section{ABSTRACT}

(Litterfall production in the Brazilian mid-western Amazonia-Cerrado transition forest). The objective of the present work was to verify the variation of litterfall production of different biomass: a cerrado ("savanna") with vegetation types Cerrado sensu stricto ("orchard-like vegetation") and Cerradão ("woodland-like vegetation") and Amazonia-Cerrado transition forest in a tropical climate. To determine the litterfall production, we used nylon screen traps. Micrometereologic data was collected in both areas of study. The litterfall in two biomass revealed themselves as seasonal, with the highest productions occurring during the dry season and the lowest during the wet. The biggest litterfall occurred in the Transition Forest, followed by the Cerrado biome. Leaf fraction was more representative than twigs, flowers, fruits in both areas studied.

KEYwORDS: Litterfall, Cerrado sensu stricto, Cerradão, Amazonia-Cerrado transition forest.

1 Universidade do Estado de Mato Grosso, Campus de Alta Floresta, Departamento de Engenharia Florestal. E-mail: cjsnx@yahoo.com.br; monicaeb@unemat.br

2 Universidade Federal de Mato Grosso, Campus de Cuiabá, Programa de Pós graduação em Física e Meio Ambiente. E-mail: Isanches@hotmail.com; f_a_lobo@yahoo.com.br; nogueira@cpd.ufmt.br. 


\section{INTROD UÇÃO}

O Brasil possui a maior área de Cerrado do Mundo, localizada em clima tropical, fazendo limites com duas florestas, a Amazônica e a Atlântica. A interação de vários fatores, incluindo o determinante fogo, resultou na presença de uma flora de grande diversidade neste ecossistema (Dias, 1992). A vegetação do Cerrado caracteriza-se por grandes variedades fitofisionômicas, dentre elas o Cerradão, com espécies ocorrentes no Cerrado e também de mata, e o Cerrado sensu stricto com árvores baixas e retorcidas, arbustos, subarbustos e ervas. Como limite, os ecossistemas florestais Amazônicos desenvolveram eficientes mecanismos de conservação de nutrientes para suportarem uma floresta (Schubart et al., 1984). Assim, a contrastante exuberância da cobertura florestal Amazônica está associada às estratégias de conservação e de reciclagem de nutrientes dentro do próprio sistema (Herrera et al., 1978; Schubart et al., 1984). Grande parte dos nutrientes dentro do ecossistema está presente na parte aérea da vegetação ocorrendo forte interação entre a vegetação e o solo, por meio da ciclagem de nutrientes, em que o acúmulo de serrapilheira exerce importante função, por ser a mais significativa forma de transferência de nutrientes (Martins \& Rodrigues, 1999).

A produtividade de um ecossistema depende da quantidade de nutrientes armazenados em seus vários compartimentos, como: a vegetação, a serrapilheira, o solo e a biomassa animal e, também, a taxa de transferência (Wetzel, 1997).

A serrapilheira é um importante componente de um ecossistema florestal compreendendo o material precipitado ao solo pela biota, o que inclui principalmente folhas, galhos, frutos, flores, raízes e resíduos animais (Dias e Oliveira Filho, 1997). Por meio do processo de decomposição, a serrapilheira libera para o solo elementos minerais que as plantas utilizam, desempenhando assim, um papel fundamental na circulação de nutrientes e nas transferências de energia entre os níveis tróficos (Ribeiro, 1998; Sioli, 1991), contribuindo para manutenção dos processos de um ecossistema (Peres et al., 1983).

Estudos sobre a produção de serrapilheira têm sido feitos por diversos autores em diferentes localidades geográficas, porém têm-se deparado com um importante obstáculo, que é a comparabilidade de dados (Dias e Oliveira Filho, 1997) por causa das diferentes metodologias aplicadas e dos distintos comportamentos dos ecossistemas. Estudos que busquem compreender a produção de serrapilheira, em diferentes biomas regidos pelo mesmo comportamento climático se tornam de grande importância para a compreensão e determinação de modelos regionais.

O presente trabalho teve como objetivo verificar a variação da produção de serrapilheira de dois biomas distintos, ambos em clima tropical: Cerrado (Cerrado sensu stricto e Cerradão) e Floresta de Transição Amazônia-Cerrado.

\section{MATERIAL E MÉTODOS}

\section{ÁREAS DE ESTUDO}

Este estudo foi desenvolvido em três áreas, sendo uma de Cerrado sensu stricto (s.s.), uma de Cerradão e uma de floresta de transição. As áreas de Cerrado s.s. e Cerradão estão localizadas na Reserva Biológica Municipal "Mário Viana", perímetro urbano de Nova Xavantina, região sudeste de Mato Grosso (14041'09”S e 52²0'09”O). A área da floresta de transição entre floresta tropical úmida e cerrado está localizada a aproximadamente $50 \mathrm{~km}$ da cidade de Sinop, região norte de

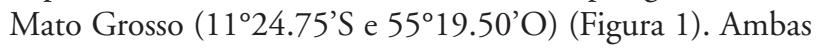
as regiōes apresentam sazonalidade climática característica, predominantemente do tipo tropical, com duas estaçóes bem definidas, sendo um período seco (maio a setembro) e um período chuvoso (outubro a abril).

\section{PRODUÇÃOO DE SERRAPILHEIRA}

As coletas foram realizadas mensalmente de abril de 2002 a maio de 2003. Para a determinação da produção de serrapilheira foram utilizados coletores confeccionados com tela de nylon de $2 \mathrm{~mm}$ de abertura de malha e com $1 \mathrm{~m}^{2} \mathrm{de}$ área. Os coletores foram colocados a $0,3 \mathrm{~m}$ acima do solo para evitar perdas de serrapilheira pela ação de microorganismos.

Na região de Cerrado foram utilizados 20 coletores, sendo dez coletores em área de Cerradão e outros 10 em área de Cerrado s.s.. Na região de floresta de transição foram utilizados 20 coletores.

As amostras coletadas foram levadas ao laboratório, onde foram separadas em fraçóes de folhas, galhos, flores e frutos e secadas em estufa até a obtenção de peso constante em balança analítica de precisão.

\section{DADOS MICROMETEOROLÓGICOS}

Para a região de Cerrado foram coletados mensalmente, junto à estação meteorológica situada na área da Reserva Biológica Municipal "Mário Viana", dados de temperatura do ar e precipitação.

O monitoramento dos fatores ambientais na regiāo da floresta de transição foi realizado por meio de equipamentos de medidas micrometeorológicas (HMP-35, Vaisala, Inc., Helsinki, Finland; 2501 Sierra-Misco, Inc., Berkeley, CA, USA) instalados numa torre meteorológica de 40 metros de altura (Vourlitis et al., 2002).

\section{ANÁLISE DOS DADOS}

Foi calculada a média mensal dos dados obtidos nos coletores de cada área e a correspondente produção anual 
de serrapilheira em cada tipo de floresta. Para analisar as relaçóes entre a umidade relativa, precipitação, temperatura e a produção de serrapilheira foi aplicada análise de correlação. A produção de serrapilheira das três áreas nos períodos de seca e chuvoso foi comparada pelo Teste t.

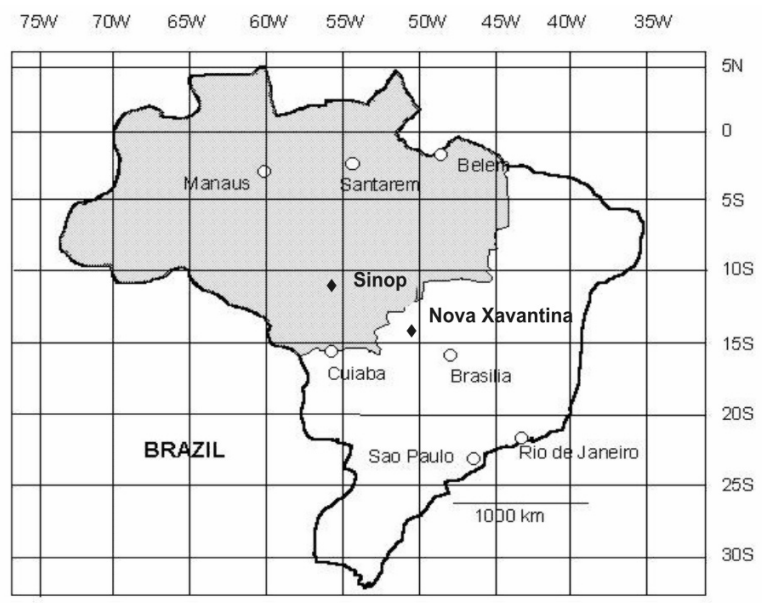

Figure 1 - Location of the study areas.

\section{RESULTADOS E DISCUSSÃO}

\section{CONDIÇÕES CLIMÁTICAS}

$\mathrm{Na}$ região de Cerrado, as maiores precipitações foram registradas no período de novembro a março e as menores entre abril e julho. A temperatura máxima foi registrada no mês de outubro e a mínima no mês de junho. $\mathrm{Na}$ área de Floresta de transição as maiores precipitaçôes foram registradas entre outubro e abril e as menores de maio a setembro. A temperatura máxima foi registrada no mês de dezembro e a mínima em agosto (Figura 2).

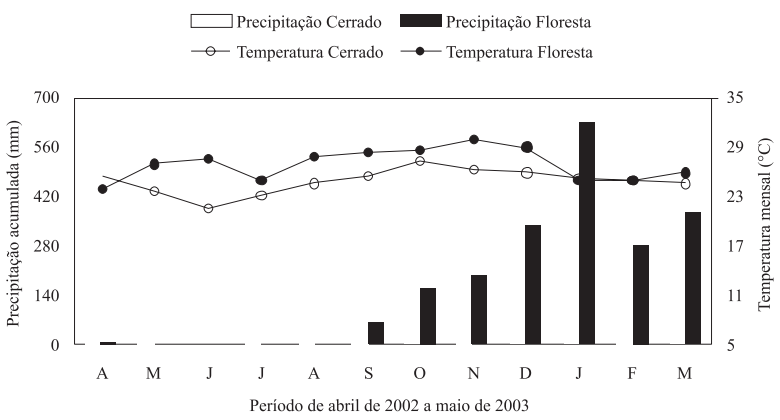

Figure 2 - Climatic data in the period from April to 2002 the March of 2003, in the Cerrado and Forest areas.

\section{PRODUÇÃO DE SERRAPILHEIRA E COMPORTAMENTO SAZONAL}

A produção média de serrapilheira no Cerrado s.s. variou de 2 a $112 \mathrm{~kg} \cdot \mathrm{ha}^{-1} \cdot \mathrm{mês}^{-1}$, resultando numa produção anual de $622 \mathrm{~kg} \cdot h \mathrm{ha}^{-1} \cdot \mathrm{a}^{-1}$. Na área de Cerradão, a produção média de serrapilheira variou de 10 a $261 \mathrm{~kg} \cdot \mathrm{ha}^{-1} \cdot \mathrm{mê}^{-1}$, com uma produção anual de $1046 \mathrm{~kg} \cdot \mathrm{ha}^{-1} \cdot \mathrm{a}^{-1}$. A produção média de serrapilheira na floresta de transição variou de 83 a 1397 $\mathrm{kg} \cdot \mathrm{ha}^{-1} \cdot \mathrm{mê}^{-1}$, atingindo uma produção anual de $6566 \mathrm{~kg} \cdot \mathrm{ha}$ ${ }^{1} \cdot \mathrm{a}^{-1}$ (Figura 3).

A maior produção de serrapilheira nas áreas estudadas foi registrada no período de seca. $\mathrm{Na}$ área de Cerrado s.s., a análise de correlação entre produção de serrapilheira, temperatura e precipitação indicou correlaçóes negativas significativas entre produção de serrapilheira e precipitação $(r=-0,815 ; p<0,05)$, indicando assim que houve uma maior deposição mensal na estação seca do que na estação úmida o que foi confirmado pelo Teste t $(\mathrm{p}=0,0002)$. Nas áreas de Cerradão e de Floresta de transição, não há correlação significativa entre produção de serrapilheira, temperatura e precipitação, e também não há diferença significativa entre os períodos de seca e chuvoso.

A produção de serrapilheira ao longo do ano foi significativamente maior na floresta de transição quando comparada as fitofisionomias do Cerrado (Cerrado s.s.: $\mathrm{p}=$ 0,001 ; Cerradão: $p=0,002$ ), enquanto que a produção entre as fitofisionomias do Cerrado não diferiu significativamente $(\mathrm{p}=0,06)$.

Dentre as fitofisionomias de Cerrado estudadas, o Cerradão apresentou, no geral, maior produção de serrapilheira. Dados semelhantes foram encontrados por Silva (1983) em um estudo feito no Cerrado do Distrito Federal. Cianciaruso et al. (2006), registrou uma produção de serrapilheira cinco vezes maior num Cerradão da Estação Ecológica de Jataí em São Paulo do que os valores registrados neste estudo.

Os valores registrados na floresta de transição foram similares aos encontrados na floresta de terra firme na Amazônia Central por Luizão e Schubart (1986) e numa floresta tropical úmida no Panamá (Wieder \& Wright, 1995).

A menor produção de serrapilheira na área de Cerrado concorda com dados obtidos por Alho (1992) que atribui essa diferença ao fato do Cerrado s.s. ser de vegetação semi-aberta com árvores espaçadas e pequenas e de ter pouca biomassa por unidade de área em relação à floresta.

A estação seca foi caracterizada pela maior produção de serrapilheira em todos os sistemas estudados. A ocorrência de maior produção de serrapilheira no período seco é influenciada principalmente pelo estresse hídrico (César, 1993; Herbohn \& Congdon, 1993), podendo ser uma resposta da vegetação que com a derrubada de folhas reduziria a perda de água por transpiração. Estudos de produção de serrapilheira realizados por Klinge \& Rodrigues (1968); Luizão \& Schubart (1987); Boinskis (1989); Dantas \& Phillipsson (1989), Luizão (1989); Morellato \& Leitão Filho (1995); Rodrigues et al. (2000); Vital et al. (2004) também observaram máxima produção de serrapilheira no período de seca. 
O aumento na queda de folhas no período de seca na área de Cerrado, segundo Barros (1979), ocorre nos meses de mais baixa precipitação, e o brotamento das folhas, a floração e a frutificação ocorrem no início e durante a estação de chuva, podendo ser influenciada por fatores ambientais variáveis como hidroperiodicidade e radiação solar.

Os ecossistemas de florestas tropicais, via de regra, apresentam produção contínua de serrapilheira no decorrer do ano, tendo a quantidade produzida nas diferentes épocas sido dependente do tipo de vegetação considerada (Leitão Filho et al., 1993) e das variações climáticas.

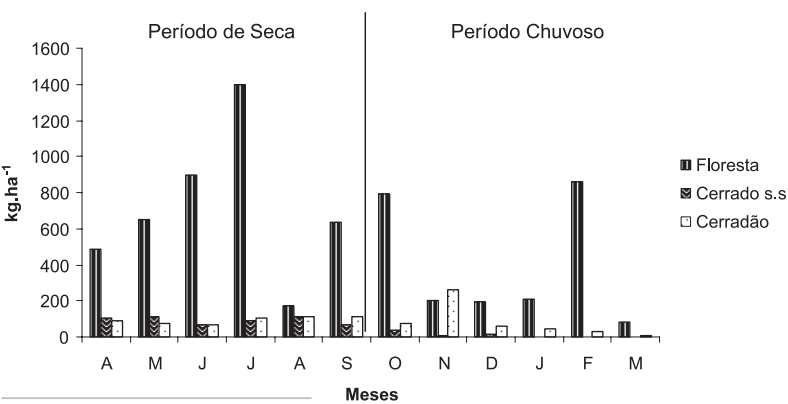

Figura 3 - Produção média mensal de serrapilheira nas áreas de Cerrado sensu stricto, Cerradão e Floresta de Transição, no período de abril/2002 a março/2003

\section{COMPOSIÇÃO DA SERRAPILHEIRA}

As folhas representam a maior parte da serrapilheira em todas as áreas estudadas, sendo ainda mais representativa na área de floresta de transição do que nas fitofisionomias de Cerrado, onde as frações frutos e galhos são mais representativas (Tabela 1 ).

As folhas representam o compartimento mais importante na maioria dos estudos de produção de serrapilheira em florestas tropicais (Barnes et al., 1998), e essa fração depende principalmente das espécies, da estrutura do local e da idade das árvores (Zimmermann et al., 2002), pois está em constante produtividade, por causa das trocas resultantes de altas taxas fotossintéticas, diferentes fenologias entre as espécies locais e pelas respostas mais rápidas às alteraçôes ambientais (Malavolta, 1989).

A elevada deposição de folhas em florestas pode ser também decorrência do rápido crescimento e renovação foliar de espécies pioneiras, canalizando os fotossimilados em um primeiro momento à produção vegetativa (Martins \& Rodrigues, 1999; Werneck et al., 2001).

O percentual de contribuição da fração foliar na serrapilheira registrado neste estudo foi semelhantemente encontrado por Klinge \& Rodrigues (1968); Luizão \& Schubart (1987); Meentmeyer et al. (1982); Pagano (1989), por Oliveira (1997), Martins \& Rodrigues (1999); Cianciaruso
Tabela 1 - Produção de serrapilheira nas áreas estudadas.

\begin{tabular}{|c|c|c|c|c|c|c|c|c|c|}
\hline \multirow{2}{*}{ FLORESTA } & \multicolumn{9}{|c|}{ Frações da serrapilheira } \\
\hline & \multicolumn{2}{|c|}{ Folhas } & \multicolumn{2}{|c|}{ Galhos } & \multicolumn{2}{|c|}{ Frutos } & \multicolumn{2}{|c|}{ Flores } & \multirow{2}{*}{ 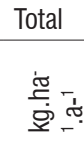 } \\
\hline Mês/Ano & 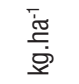 & ๙0 & 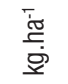 & ه̊ & $\begin{array}{l}\bar{c} \\
\overline{\underline{c}} \\
\bar{g}\end{array}$ & ه̊ & 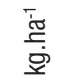 & o & \\
\hline Abril/2002 & 391 & 81 & 78 & 16 & 0 & 0 & 15 & 3 & 484 \\
\hline Maio/2002 & 548 & 84 & 91 & 14 & 0 & 0 & 13 & 2 & 652 \\
\hline Junho/2002 & 767 & 85 & 114 & 13 & 0 & 0 & 18 & 2 & 899 \\
\hline Julho/2002 & 1305 & 93 & 78 & 6 & 0 & 0 & 15 & 1 & 1397 \\
\hline Agosto/2002 & 120 & 71 & 19 & 11 & 0 & 0 & 30 & 18 & 168 \\
\hline Setembro/2002 & 578 & 91 & 54 & 9 & 0 & 0 & 0 & 0 & 632 \\
\hline Outubro/2002 & 789 & 100 & 0 & 0 & 0 & 0 & 0 & 0 & 789 \\
\hline Novembro/2002 & 200 & 100 & 0 & 0 & 0 & 0 & 0 & 0 & 200 \\
\hline Dezembro/2002 & 182 & 93 & 7 & 4 & 0 & 0 & 6 & 3 & 195 \\
\hline Janeiro/2003 & 128 & 62 & 47 & 22 & 0 & 0 & 33 & 16 & 207 \\
\hline Fevereiro/2003 & 475 & 55 & 251 & 29 & 0 & 0 & 134 & 16 & 859 \\
\hline Março/2003 & 64 & 77 & 8 & 9 & 0 & 0 & 11 & 13 & 83 \\
\hline Total & 5547 & & 746 & & 0 & & 274 & & 6566 \\
\hline \multicolumn{10}{|l|}{ CERRAD0 s.s. } \\
\hline Abril/2002 & 12 & 11 & 13 & 12 & 80 & 74 & 2 & 2 & 107 \\
\hline Maio/2002 & 34 & 31 & 10 & 9 & 67 & 60 & 0 & 0 & 111 \\
\hline Junho/2002 & 38 & 60 & 12 & 19 & 14 & 21 & 0 & 0 & 64 \\
\hline Julho/2002 & 60 & 65 & 20 & 21 & 12 & 13 & 0 & 0 & 92 \\
\hline Agosto/2002 & 68 & 61 & 39 & 35 & 0 & 0 & 5 & 4 & 112 \\
\hline Setembro/2002 & 28 & 43 & 16 & 24 & 0 & 0 & 22 & 33 & 66 \\
\hline Outubro/2002 & 15 & 39 & 0 & 0 & 14 & 36 & 10 & 25 & 38 \\
\hline Novembro/2002 & 7 & 63 & 0 & 0 & 2 & 19 & 2 & 17 & 10 \\
\hline Dezembro/2002 & 2 & 11 & 1 & 6 & 12 & 78 & 1 & 5 & 15 \\
\hline Janeiro/2003 & 1 & 42 & 1 & 50 & 0 & 0 & 0 & 8 & 2 \\
\hline Fevereiro/2003 & 2 & 71 & 0 & 14 & 0 & 0 & 0 & 14 & 2 \\
\hline Março/2003 & 1 & 21 & 1 & 38 & 1 & 34 & 0 & 7 & 3 \\
\hline Total & 267 & & 112 & & 201 & & 42 & & 622 \\
\hline \multicolumn{10}{|l|}{ CERRADÃO } \\
\hline Abril/2002 & 52 & 56 & 38 & 42 & 0 & 0 & 2 & 2 & 92 \\
\hline Maio/2002 & 48 & 61 & 29 & 37 & 0 & 0 & 2 & 2 & 78 \\
\hline Junho/2002 & 59 & 85 & 8 & 11 & 0 & 0 & 3 & 4 & 70 \\
\hline Julho/2002 & 85 & 83 & 13 & 12 & 0 & 0 & 5 & 5 & 102 \\
\hline Agosto/2002 & 92 & 83 & 9 & 8 & 0 & 0 & 10 & 9 & 111 \\
\hline Setembro/2002 & 64 & 59 & 25 & 23 & 0 & 0 & 20 & 18 & 109 \\
\hline Outubro/2002 & 19 & 25 & 18 & 25 & 9 & 12 & 28 & 38 & 74 \\
\hline Novembro/2002 & 14 & 5 & 218 & 84 & 10 & 4 & 20 & 8 & 261 \\
\hline Dezembro/2002 & 11 & 17 & 0 & 0 & 22 & 36 & 29 & 47 & 62 \\
\hline Janeiro/2003 & 20 & 43 & 19 & 39 & 8 & 17 & 1 & 1 & 48 \\
\hline Fevereiro/2003 & 18 & 64 & 6 & 20 & 5 & 16 & 0 & 0 & 29 \\
\hline Março/2003 & 9 & 85 & 1 & 10 & 1 & 5 & 0 & 0 & 10 \\
\hline Total & 489 & & 384 & & 54 & & 119 & & 1046 \\
\hline
\end{tabular}


et al. (2006). Observa-se na região de floresta que no mês de janeiro houve a máxima precipitação mensal e em fevereiro de 2003 houve um aumento da produção de folhas, isso pode ser decorrente dos fenômenos climáticos que forçam o desprendimento de parte da vegetação, provocado pela ação mecânica de ventos e chuvas fortes.

Houve, nas áreas de Cerrado e Cerradão, uma maior tendência da floração no final da estação seca e início da estação úmida como também observado por Dias e Oliveira - Filho (1996), concordando com o estudo de Morellato \& Leitão Filho (1995) sobre as fenofases na floresta de Santa Genebra localizada em Campinas - SP, com floração no final da estação seca e início da estação úmida, chegando a um mínimo no inverno. Jackson (1978) encontrou em uma floresta umbrófila submontana em Santa Teresa-ES, um padrão de sazonalidade semelhante, com um maior número de espécies florindo do final da estação seca ao início da estação chuvosa, e afirma que esse padrão é bem típico de florestas tropicais.

O percentual de contribuição da fração frutos na serrapilheira para área de Cerradão ficou próximo aos percentuais encontrados por Pagano (1989), em florestas semidecídua de Rio Claro, SP, por Sanchez \& Alvarez-Sanchez (1995), em floresta secundária do México, e por Martins e Rodrigues (1999), em clareiras de floresta semidecídua de Campinas, SP.

Segundo Wheit (1994), o padrão de queda de frutos na Reserva de Lopé, no Gabão, depende do modo de dispersão da espécie, mas parece haver uma tendência de baixa produção na época da seca, com um ou mais picos na estação chuvosa, indicando que possivelmente sua deposição seja dependente da estratégia de dispersão adotada pela espécie para sua reprodução e não esteja em função das variações do microclima. Para as áreas estudadas, esta teoria foi confirmada na área de Cerradão a qual apresentou baixa produção na estação seca e alguns picos na estação de chuva. Entretanto, no Cerrado s.s. ocorreu o inverso. Isto acontece porque o Cerrado possui espécies precoces e tardias fazendo com que produza frutos em quase todos os meses do ano (Felfili \& Silva Junior, 2001).

Em estudos conduzidos em florestas paulistas por Carpanezzi (1980) e Pagano (1989), foi registrada contribuição da fração galhos na serrapilheira total semelhante ao registrado neste. Segundo Ribeiro (1998), a produção da fração galhos pode estar relacionada à incidência de ventos no local.

\section{CONCLUSÕES}

A produção de serrapilheira nas áreas em estudo mostrouse sazonal, com a maior queda de serrapilheira ocorrendo durante a estação seca e a menor durante a estação chuvosa, nos três ecossistemas. A maior produção anual de serrapilheira ocorreu na Floresta de Transição, seguida pelo Cerradão. A fração foliar representou $2 / 3$ ou mais do total da serrapilheira, em todos os ecossistemas, enquanto que a fração frutos teve uma maior produção no Cerrado sensu stricto do que no Cerradão e na floresta de transição.

\section{AGRADECIMENTOS}

Ao Projeto LBA (Experimento de Grande Escala da Biosfera-Atmosfera na Amazônia), por fomentar a pesquisa na área de transição Amazônia-Cerrado; à Universidade Federal de Mato Grosso e à Universidade do estado de Mato Grosso, pela disponibilização dos laboratórios.

\section{BIBLIOGRAFIA CITADA}

Alho, C.J.R. 1992. A teia da vida: uma introdução á ecologia brasileira. Editora Objetiva, Rio de Janeiro. 160pp.

Barnes, B.V.; Zak, D.R.; Denton, S.R.; Spurr, S.H. 1998. Forest Ecology. Editora John Wiley, New York. 774pp.

Barros, M. 1979. Variação de diâmetro em árvores do Cerrado relacionada à fenologia e aos fatores ambientais. Dissertação de Mestrado, Universidade de Brasília, Distrito Federal. 184pp.

Boinskis, S. 1989. Seasonal patterns in a tropical lowland forest. Biotropica, 21(33): 223-233.

Carpanezzi, A.A. 1980. Deposição de material orgânico e nutrientes em uma floresta natural e em uma plantação de Eucalyptus no interior do Estado de São Paulo. Dissertação de Mestrado. ESALQ/USP, Piracicaba, São Paulo. 107pp.

César, O. 1993. Produção de serrapilheira na mata mesófila semidecídua da Fazenda Barreiro Rico, município de Anhembi, SP. Revista Brasileira de Biologia, 53: 671-681.

Cianciaruso, M.C.; Pires, J.S.R.; Delitti, W.B.C.; Silva, E.F.L.P. 2006. Produção de serrapilheira e decomposição do material foliar em um cerradão na Estação Ecológica de Jataí, município de Luiz Antônio, SP, Brasil. Acta Bot. Bras., 20(1): 49-59.

Dantas, M.; Phillipson, J. 1989. Litterfall and litter nutrient content in primary and secundary Amazonian "Terra firme" rain forest. Journal of Tropical Ecology, 5: 27-36.

Dias, B.F.S. 1992. Alternativas de desenvolvimento dos cerrados: manejo e conservação dos recursos naturais renováveis. Fundação Pró-Natureza, Brasília, Distrito Federal. 97pp.

Dias, H.C.T.; Oliveira Filho, A.T. 1997. Variação temporal e espacial da produção de serrapilheira em uma área de floresta estacional semidecídua montana em Larvras-MG. Revista Árvore, 21: 11-26.

Dias, H.C.T.; Oliveira Filho, A.T. 1996. Fenologia de quatro espécies arbóreas de uma floresta estacional semidecídua montana em Lavras-MG. Revista Cerne, 2: 38-42.

Felfili, J.M.; Silva Junior, M.C. 2001. Biogeografia do Bioma Cerrado. Editora UNB, Brasilia. 152pp.

Herbohn, J.L.; Congdon, R.A. 1993. Ecosystem dynamics disturbed and undisturbed sites in north Queensland wet tropical rain forest. II - Litterfall. Journal of Tropical Ecology, 9: 365-380. 
Herrera, R.; Jordan, C.F.; Klinge, H.; Medina, E. 1978. Amazon ecosystems: Their structure and functioning with particular emphasis on nutrients. Interciencia, 3: 223-232.

Jackson, J.F. 1978. Seazonality of flowering and leaf-fall in a Brazilian subtropical lower montane moist forest. Biotropica, 10: 38-42.

Klinge, H.; Rodrigues, W.A. 1968. Litter production in an area of Amazonian terra firme forest. Part.I: Litter-fall, organic carbon and total nitrogen contents of litter. Amazoniana, 1 (4): $287-$ 302.

Leitão Filho, H.F.; Pagano, S.N.; Cesar, O.; Timoni, J.L.; Rueda, J.J. 1993. Ecologia da mata atlântica em Cubatão, SP. EDUNESP/ EDUNICAMP, São Paulo. 86pp.

Luizão, F.J.; Schubart, H.O.R. 1986. Produção e decomposição de liteira em floresta de terra firme da Amazônia Central. Acta Limnol. Bras. 1: 575-600.

Luizão, F.J.; Schubart, H.O.R. 1987. Litter production and decomposition in a terra-firme forest of Central Amazonia. Experientia, 43(3): 259-265.

Luizão, F.J. 1989. Litter production and mineral element input to the forest floor in a Central Amazonian forest. GeoJournal, 19(4): 407-417.

Malavolta, E. 1989. Avaliação do estado nutricional das plantas: princípios e aplicaçôes. Associação Brasileira para Pesquisa do Potássio e do Fosfato. Piracicaba, São Paulo. 201pp.

Martins, S.V.; Rodrigues, R.R. 1999. Produção de serrapilheira em clareiras de uma floresta estacional semidecidual no Município de Campinas, SP. Revista Brasileira de Botânica, 22 (3): 405-412.

Meentmeyer, V.; Box, E.O.; Thompson, R. 1982. World patterns and amounts of terrestrial plant litter production. BioScience, 32: $125-128$.

Morellato, P.C.; Leitão Filho, H.F. 1995. Ecologia e preservação de uma floresta tropical urbana: reserva de Santa Genebra. UNICAMP, Campinas, São Paulo. 79pp.

Oliveira, R.E. 1997. Aspectos da dinâmica de um fragmento florestal em Piracicaba-SP: silvigênese e ciclagem de nutrientes. Dissertação de Mestrado. ESALQ/USP, Piracicaba, São Paulo. 85pp.

Pagano, S.N. 1989. Produção de folhedo em mata mesófila semidecídua no Município de Rio Claro, São Paulo. Revista Brasileira de Biologia, 1: 634-639.

Peres, J.R.R.; Suhet, A.; Vargas, M.A.T.; Drozdowicz, A. 1983. Litter production in areas of Brazilian "Cerrados". Pesquisa Agropecuária Brasileira, 18(9): 1037-43.

Ribeiro, L. 1998. Dinâmica de nutrientes na serrapilheira, em um trecho de mata ciliar alagável com ninhal de aves do Rio Cuiabá, no Pantanal Barão de Melgaço-MT. Monografia de graduação. Universidade Federal de Mato Grosso, Instituto de Biociências, Cuiabá, Mato Grosso. 53pp.
Rodrigues, W.A.; Klinge, H.; Fittkau, E.J. 2000. Estrutura e funcionamento de um ecossistema florestal amazônico de terra firme junto à Reserva Florestal Walter Egler, município de Rio Preto da Eva, Amazonas, Brasil. Acta Biol. Par., 29(1, 2, 3, 4): 219-243.

Sanchez, G.; Alvarez-Sanchez, J. 1995. Litterfall in primary and secondary tropical forests of Mexico. Tropical Ecology, 36: 191-201.

Schubart, H.; Franken, W.; Luizão, F. 1984. Uma floresta sobre solos pobres. Ciência Hoje, 2(10): 26-32.

Silva, I.S. 1983. Alguns Aspectos de Ciclagem de Nutrientes em uma área de Cerrado: Chuva, Produção e Decomposição do Litter. Dissertação de Mestrado. Universidade de Brasília, Brasília, Distrito Federal. 127pp.

Sioli, H. 1991. Amazônia: fundamentos da ecologia da maior região de florestas tropicais. Editora Vozes, Petrópolis, Rio de Janeiro. 72pp.

Vital, A.R.T.; Guerrini, I.G.; Franken, W.K.; Fonseca, R.C.B. 2004. Produção de serrapilheira e ciclagem de nutrientes de uma floresta estacional semidecidual em zona ripária. Revista Árvore, 28(6): 793-800.

Vourlitis, G.L.; Priante Filho, N.; Hayashi, M.M.S.; Sousa Nogueira, J.; Caseiro, F.T.; Campelo Jr, H.J. 2002. Seasonal variations in the evapotranspiration of a transitional tropical forest of Mato Grosso, Brazil. Water Resources Research, 38(6): 30-42.

Werneck, M.S.; Pedralli, G.; Gieseke, L.F. 2001. Produção de serrapilheira em três trechos de uma floresta semidecídua com diferentes graus de perturbação na Estação Ecológica do Tripuí, Ouro Preto. Revista Brasileira de Botânica, 24(2): 195-1998.

Wetzel, M.M.V.S. 1997. Época de dispersão e fisiologia de sementes do Cerrado. Tese de Doutorado. Universidade de Brasília, Brasília, Distrito Federal. 175pp.

Wheit, L.J.T. 1994. Patterns of fruit-fall phenology in the Lopé Reserv, Gabon. Journal of Tropical Ecology, 10: 289-312.

Wieder, K.; Wright, J.S. 1995. Tropical forest litter dynamics and dry season irrigation on Barro Colorado island, Panama. Ecology, 76(6): 1971-1979.

Zimmermann, S.; Braun, S.; Conedera, M.; Blaser, P. 2002. Macronutrient inputs by litterfall as opposed to atmospheric deposition into two contrasting chestnut forest stands in southern Switzerland. Forest Ecology and Management, 161: 289-302.

Recebido em 22/01/2007

Aceito em 04/09/2007 